\title{
Safety, pharmacokinetics, and pharmacodynamics of oral omaveloxolone (RTA 408), a synthetic triterpenoid, in a first-in-human trial of patients with advanced solid tumors
}

\author{
This article was published in the following Dove Press journal: \\ OncoTargets and Therapy \\ 29 August 2017 \\ Number of times this article has been viewed
}

\section{Ben C Creelan' \\ Dmitry I Gabrilovich ${ }^{2}$ \\ Jhanelle E Gray' \\ Charles CWilliams' \\ Tawee Tanvetyanon' \\ Eric B Haura' \\ Jeffrey SWeber ${ }^{3}$ \\ Geoffrey T Gibney ${ }^{4}$ \\ Joseph Markowitz ${ }^{5}$ \\ Joel W Proksch ${ }^{6}$ \\ Scott A Reisman ${ }^{6}$ \\ Mark D McKee ${ }^{7}$ \\ Melanie P Chin ${ }^{6}$ \\ Colin J Meyer ${ }^{6}$ \\ Scott J Antonia' \\ 'Department of Thoracic Oncology, H. Lee Moffitt Cancer Center and Research Institute, Magnolia Drive, Tampa, FL, USA; ${ }^{2}$ The Wistar Institute, Philadelphia, PA, USA; ${ }^{3}$ Laura and Isaac Perlmutter Cancer Center, New York, NY, USA; ${ }^{4}$ Department of Medicine, Georgetown-Lombardi Comprehensive Cancer Center, Washington, DC, USA; ${ }^{5}$ Department of Cutaneous Oncology, H. Lee Moffitt Cancer Center and Research Institute, Magnolia Drive, Tampa, FL, USA; ${ }^{6}$ Reata Pharmaceuticals, Inc., Irving, TX, USA; ${ }^{7} A b b V i e$, Inc., North Chicago, IL, USA}

Correspondence: Ben C Creelan H. Lee Moffitt Cancer Center, 12902 Magnolia Drive, Tampa, FL 336I2, USA $\mathrm{Tel}+$ I 8 I 37453050

Fax + I 8I37453027

Email ben.creelan@moffitt.org
Background: Omaveloxolone is a semisynthetic oleanane triterpenoid that potently activates Nrf2 with subsequent antioxidant function. We conducted a first-in-human Phase I clinical trial (NCT02029729) with the primary objectives to determine the appropriate dose for Phase II studies, characterize pharmacokinetic and pharmacodynamic parameters, and assess antitumor activity.

Methods: Omaveloxolone was administered orally once daily continuously in a 28-day cycle for patients with stage 4 relapsed/refractory melanoma or non-small cell lung cancer. An accelerated titration design was employed until a grade 2-related adverse event (AE) occurred. A standard $3+3$ dose escalation was employed. Single-dose and steady-state plasma pharmacokinetics of the drug were characterized. Downstream Nrf2 activation was assessed in peripheral blood mononuclear cells by quantification of target gene mRNA expression.

Results: Omaveloxolone was tested at four dose levels up to $15 \mathrm{mg}$ given orally once daily. No dose-limiting toxicities were detected, and the maximum tolerated dose was not determined. All drug-related AEs were either grade 1 or 2 in severity, and none required clinical action. The most common drug-related AEs were elevated alkaline phosphatase (18\%) and anemia (18\%). No drug interruptions or reductions were required. Omaveloxolone was rapidly absorbed and exhibited proportional increases in exposure across dose levels. With some exceptions, an overall trend toward time-dependent and dose-dependent activation of Nrf2 antioxidant genes was observed. No confirmed radiologic responses were seen, although one lung cancer subject did have stable disease exceeding 1 year.

Conclusions: Omaveloxolone has favorable tolerability at biologically active doses, although this trial had a small sample size which limits definitive conclusions. These findings support further investigation of omaveloxolone in cancer.

Keywords: antioxidant inflammation modulator, nitrotyrosine, nitric oxide synthase, melanoma, non-small cell lung cancer, immuno-oncology, myeloid-derived suppressor cells, bardoxolone methyl

\section{Background}

Despite progress in T-cell immunotherapy, antigen-specific tolerance caused by MDSCs remains a problematic mechanism of tumor cell resistance. ${ }^{1}$ MDSCs facilitate peripheral immunosuppression via multiple mechanisms, including production of ROS thereby suppressing CD8 ${ }^{+}$T-cell cytolytic function. ${ }^{2,3}$ Synthetic triterpenoids are a subclass of antioxidant inflammation modulators, derived from oleananes found 
in medicinal plants. This class of compounds reduces the production of reactive oxygen and nitrogen species and also inhibits MDSC-mediated suppression of effector T-cells. ${ }^{4}$ Triterpenoids bind at the specific cysteine residue cys 151 on Kelch-like ECH-associated protein 1, thereby releasing the transcription factor Nrf2 into the nucleus., ${ }^{5,6}$ Activation of Nrf2 causes expression of downstream antioxidant genes in a coordinated cellular pathway, thereby protecting cells against oxidative and electrophilic stress. Induction of Nrf2 also has anti-inflammatory effects via inhibition of ROSmediated inflammatory signaling pathways. $^{7}$

Omaveloxolone (RTA 408) is a semisynthetic triterpenoid developed through lead optimization to suppress interferon$\gamma$-mediated nitric oxide signaling in macrophages, even at nanomolar concentrations. ${ }^{8}$ The drug suppresses reactive oxygen and NT production in spleen-derived MDSCs and tumor xenografts. ${ }^{8}$ At higher concentrations, omaveloxolone selectively inhibits tumor cell growth by blockade of inhibitor of nuclear factor kappa-B kinase subunit. ${ }^{8}$ Similarly, omaveloxolone can promote apoptosis of established murine cancer xenografts. ${ }^{9}$ Administration of systemic omaveloxolone also protects hematopoietic cell function in lethally irradiated mice. ${ }^{10}$ Furthermore, topical application of omaveloxolone causes dose-proportional induction of Nrf2-target genes in the skin of rats, and in punch biopsies in human volunteers. ${ }^{11-13}$ Along these lines, pretreatment with omaveloxolone also protects retinal pigment epithelial cells from oxidative destruction via activation of Nrf2. ${ }^{14}$ Based on in vitro and animal studies, omaveloxolone exhibited high protein binding with extensive tissue distribution, making it a suitable potential oncologic agent.

Based on robust preclinical antitumor activity of omaveloxolone and related analogs, we sought to test the drug in human subjects. ${ }^{8}$ Therefore, we conducted a first-in-human Phase I trial, with the primary objective to characterize the safety and pharmacokinetics of omaveloxolone as an oral capsule. Our hypothesis was that omaveloxolone would be safe and well tolerated in human subjects.

\section{Methods}

\section{Study design}

This was an open-label, single-center, Phase I study using a modified 3+3 dose-escalation design (NCT02029729). During dose escalation, the dose of omaveloxolone was to be doubled until drug-related toxicity of grade $\geq 2$ was observed. Dose escalations from this point would be limited to $\leq 50 \%$ of the previous dose (if grade $\leq 2$ ) or $33 \%$ in the event of grade $\geq 3$ toxicities.
Omaveloxolone was administered starting on day 1 , on a continuous once-daily oral schedule with a cycle of 28 days. For this first-in-human study, the recommended starting dose in humans of $2.5 \mathrm{mg}$ once daily was chosen based on the available preclinical pharmacokinetic data, the concentrations observed to modulate Nrf2 in vitro, and on the no observed adverse effect levels in rodent and monkey species from toxicity studies.

This trial was conducted at the H. Lee Moffitt Cancer Center (Tampa, FL, USA), after approval by institutional review board, Liberty IRB, federal wide registration \#008679, study \#13.11.0015. It was performed in accordance with the Declaration of Helsinki and the $I C H-G C P$ guidelines. Informed consent was obtained from all patients. The primary objectives were to characterize the safety and tolerability of a once-daily oral administration, determine the recommended Phase II dose, pharmacokinetics, pharmacodynamics, and preliminary clinical activity of omaveloxolone.

\section{Eligibility}

Patients aged 18 years or older, with histologically confirmed stage 4 NSCLC or melanoma who had experienced disease progression after more than one standard therapy were eligible. Other inclusion criteria included: BNP $<100 \mathrm{pg} / \mathrm{mL}$, toxicity from prior therapy resolved to grade $\leq 1$, an Eastern Cooperative Oncology group performance status $\leq 2$, life expectancy $\geq 12$ weeks, and adequate organ function. Significant exclusion criteria included: QTc interval on electrocardiogram (ECG) at screening of $>450$ mseconds for males and $>460$ mseconds for females. Based on preliminary in vitro data, patients taking substrates for p-glycoprotein, organic anion transporting polypeptide 1B3 (OATP1B3), organic anion transporter 1 (OAT1), CYP2C8, or CYP3A4 were also excluded. Patients with stable, treated brain metastases were eligible.

\section{Definitions of DLT and MTD}

Dose-limiting toxicity (DLT) was based on toxicities observed in the first cycle and assessed by the investigator as possibly related to omaveloxolone. A DLT was defined as any hepatobiliary disorders grade $\geq 2$, or any grade $\geq 3$ non-hepatobiliary toxicity of any duration. Grade 3 nausea or vomiting were considered as DLTs only if they occurred despite optimal medical management. Total bilirubin, aspartate aminotransferase, and alanine aminotransferase were considered DLTs if $>8 \times$ upper limit of normal $(\mathrm{ULN}),>5 \times \mathrm{ULN}$ for over 2 weeks, or $>3 \times$ ULN with symptoms. The MTD was defined as the highest dose at 
which $\leq 1$ of six DLTs $(<33 \%)$ patients experienced DLT at that dose level.

\section{Safety and efficacy}

Clinical and laboratory assessments were conducted at baseline and weekly thereafter. Safety assessments included medical history, physical examination, ECG, hematology, urinalysis, and biochemistry including BNP. QT interval was corrected using Fridericia formula. Adverse events (AEs) were graded using CTCAE version 4.0. Tumor assessments were performed every two cycles using RECIST guideline version 1.1. ${ }^{15}$ Patients were followed up for survival and toxicity for at least 30 days after drug discontinuation.

\section{Pharmacokinetics}

Plasma levels of omaveloxolone were determined from blood samples collected on day 1: pre-dose, 1, 2, 3, 4, 8 , and 24 hours after dose administration; and on day 28: pre-dose, $1,2,3,4,6,8,10$, and 24 hours after dose administration. Plasma levels were then collected on day 28 of every cycle thereafter. Plasma was separated and stored at $-70^{\circ} \mathrm{C}$ until analysis. Pharmacokinetic samples were analyzed using a validated liquid chromatographytandem mass spectrometry quantitation method by Abbvie, Inc. (North Chicago, IL, USA). Standard pharmacokinetic parameters were determined using a noncompartmental method with WinNonlin ${ }^{\mathrm{TM}}$ software version 6.3 (Pharsight Corp., Cary, NC, USA). Nominal sample collection times were used in the analysis. Parameters included the $C_{\max }$, $T_{\max }$, and $t^{1 / 2}$. The area under the plasma concentrationtime curve from time zero to the time $(t)$ of the last measurable concentration $\left(\mathrm{AUC}_{0-t}\right)$ was determined using the linear $\log$ trapezoidal rule. The apparent terminal half-life was obtained as $0.693 / \lambda z$, where $\lambda z$ represents the terminal elimination rate constant. One patient in the $2.5 \mathrm{mg}$ cohort was inadvertently dosed prior to their 24-hour collection and this sample was excluded. One sample tube in the $10 \mathrm{mg}$ cohort was received broken and could not be analyzed.

\section{Nrf2 pathway alteration biomarkers}

PBMCs were isolated from blood collected at each timepoint. Day 28 collections were missing for the $15 \mathrm{mg}$ cohort due to patient refusing blood draw (1), and due to missed visit (1). mRNA was quantified using Quantigene Plex 2.0 technology according to manufacturer's protocol (Affymetrix Inc., Santa Clara, CA, USA) and as previously described. ${ }^{13}$ Probe sets were designed against the human genome for analysis of Nrf2 target genes; a modified version of Panel 11834 (Affymetrix) was used. Genes were normalized to the mean expression of housekeeping genes RPL13A (60S ribosomal protein L13a) and peptidyl-prolyl cis-trans isomerase B.

Peripheral blood cells were quantified by automated cell counter using flow cytometry for white blood cell differential (Sysmex, XN9000).

\section{Validation of research antibodies}

Slides were stained using a Ventana Discovery XT automated system (Ventana Medical Systems, Tucson, AZ, USA) as per manufacturer's protocol with proprietary reagents. Slides were deparaffinized on the automated system with EZ Prep solution. Levels of inducible isoform of nitric oxide synthase (iNOS) and NT protein in tumor sections were determined by immunohistochemistry (IHC) using polyclonal rabbit anti-NOS2 (Abcam, Cambridge, MA, USA; ab3523) and mouse monoclonal anti-NT (Millipore, Temecula, CA, USA; clone 2A8.2).

For iNOS, optimization was performed with Protease 1 (Ventana) enzymatic retrieval method, using mouse epithelial lung tissue as a positive control. A rabbit primary antibody for iNOS (ab3523; Abcam) was incubated (1:200) in antibody diluent (Dako, Carpenteria, CA, USA) for 32 minutes. The Ventana OmniMap anti-rabbit secondary antibody was incubated for 16 minutes, followed by detection (Ventana ChromoMap kit), and slides were then counterstained with hematoxylin. Slides were then dehydrated and coverslipped as per normal laboratory protocol.

For NT, heat-induced antigen retrieval method was used (Ribo-CC, \#760-107; Ventana). Optimization was performed using breast epithelial tumor specimens as a positive control. The mouse monoclonal antibody that reacts to NT (\#MAB5404; Millipore) was incubated (1:400) in antibody diluent (Dako) for 60 minutes, followed by anti-mouse secondary antibody (Ventana) for 8 minutes.

\section{Analysis of tumor biopsies}

Tumor biopsies were not required as part of the study. However, serial archival tumor samples, collected before and during omaveloxolone treatment, of two patients with metastatic melanoma were available. Tissues were collected and preserved in formalin. Suitable areas were selected by a pathologist and sectioned into $5 \mu \mathrm{m}$ sections placed on slides. Levels of iNOS and NT protein in tumor sections were determined by IHC using polyclonal rabbit anti-NOS2 (Abcam; ab3523) and mouse monoclonal anti-NT (Millipore; clone 2A8.2). IHC was performed with Dako Link 48 autostainer after high $\mathrm{pH}$ heat-induced epitope retrieval digestion. 
IHC was performed with appropriate positive and negative controls. Slides at $4 \times$ magnification were assessed by an independent pathologist blinded to collection time, using a qualitative intensity scale of negative, weak, moderate, or strong cytoplasmic staining. A quantitative scoring method was not possible due to scant tumor material.

From those individuals with sufficient available archival tumor tissue, gene mutational analysis was carried out on DNA extracted from archival tumor samples using PyroMark $^{\circledR}$ PCR or Sequenom MassARRAY OncoCarta ${ }^{\circledR}$ platform according to the manufacturer's protocol.

\section{Statistical analysis}

Categorical variables were compared using Fisher's exact test, and Student's $t$-test was used for continuous variables. Where appropriate, changes between timepoints, QTc, and BNP measurements over time were assessed using Wilcoxon signed-rank test. Median survival was estimated by KaplanMeier method and CIs were reported. Statistical analysis was performed using Prism 6.0 (La Jolla, CA, USA) and SPSS 17.0 (SPSS Inc., Chicago IL, USA) software. Nrf2 target gene data were analyzed with Sigmaplot 12.0 (Systat, Inc., San Jose, CA, USA).

\section{Results}

\section{Patient characteristics}

Of 19 patients screened at a single institution between 2014 and 2015, 11 were eligible and treated. All patients had received several prior therapies and had progressive, measurable disease (Table 1). All patients had significant disease burden with multiple metastases. All melanoma patients had received prior anti CTLA-4 and anti-PD-1 therapies.

\section{Treatment and dose escalation}

Dose escalation proceeded through four dose levels up to $15 \mathrm{mg}$. No DLTs were observed, and the MTD was not determined. A decision was reached to stop the $15 \mathrm{mg}$ dose level in two subjects due to activation of a subsequent doseescalation trial evaluating omaveloxolone in combination with checkpoint inhibitor therapy; NCT02259231. Subjects were required to complete a mandatory DLT evaluation period of $\geq 28$ days and all were included in the safety analysis.

\section{Safety and tolerability}

Subjects received a mean of 87 days of continuous oral drug exposure. Omaveloxolone was well tolerated up to $15 \mathrm{mg}$, and all drug-related AEs were mild or moderate in severity (Table 2). The most common drug-related AEs
Table I Patient characteristics

\begin{tabular}{|c|c|}
\hline Characteristic & Total $(n=I I)$ \\
\hline \multicolumn{2}{|l|}{ Age, years } \\
\hline Median (range) & $59.0(43-78)$ \\
\hline \multicolumn{2}{|l|}{ Gender, n (\%) } \\
\hline Female & $4(36)$ \\
\hline \multicolumn{2}{|l|}{ Race, n (\%) } \\
\hline Caucasian & $10(91)$ \\
\hline Black & I (9) \\
\hline \multicolumn{2}{|l|}{ Ethnicity, n (\%) } \\
\hline Non-Hispanic & II $(100)$ \\
\hline \multicolumn{2}{|l|}{ Cancer type, n (\%) } \\
\hline Lung, adenocarcinoma & $5(45)$ \\
\hline Lung, squamous uveal & $3(27)$ \\
\hline Melanoma & $3(27)$ \\
\hline \multicolumn{2}{|l|}{ AJCC stage, $n(\%)$} \\
\hline IV & II (I00) \\
\hline \multicolumn{2}{|l|}{$\mathrm{BMI}, \mathrm{mg} / \mathrm{m}^{2}$} \\
\hline Mean (SD) & $27.7(4.2)$ \\
\hline \multicolumn{2}{|l|}{ Albumin, g/dL } \\
\hline Mean (SD) & $4.1(0.5)$ \\
\hline \multicolumn{2}{|c|}{ Prior PD-I or PD-LI mAb, n (\%) } \\
\hline Yes & $6(55)$ \\
\hline No & $5(45)$ \\
\hline \multicolumn{2}{|l|}{ Cohort, mg; n (\%) } \\
\hline 2.5 & $3(27)$ \\
\hline 5 & $3(27)$ \\
\hline 10 & $3(27)$ \\
\hline $15^{*}$ & $2(18)$ \\
\hline \multicolumn{2}{|c|}{ Prior lines of systemic therapy } \\
\hline Median, range & $4(2-7)$ \\
\hline \multicolumn{2}{|l|}{ Prior radiation, $\mathrm{n}(\%)$} \\
\hline Yes & $8(73)$ \\
\hline No & $3(27)$ \\
\hline \multicolumn{2}{|c|}{ Prior brain metastases, n (\%) } \\
\hline Yes & $5(45)$ \\
\hline No & $6(55)$ \\
\hline
\end{tabular}

Notes: All percentages are rounded and therefore may not exactly sum to one. Stage is assessed at the date of treatment. *Dose level completed in two subjects. Abbreviations: AJCC, American Joint Committee on Cancer; BMI, body mass index; mAb, monoclonal antibody; PD-I, programmed cell death protein I.

were elevated alkaline phosphatase and anemia. The most common AE regardless of causality was pain (54\%) and anemia (45\%) (Table 3). No drug-related AEs required any clinical action by the investigators. There was no detectable difference in the toxicity profile between the assessed dose levels. No drug interruptions were required. One subject with squamous lung cancer at the $15 \mathrm{mg}$ dose level had emergence of dyspnea with multifocal new nodules identified on CT of the thorax. However, subsequent imaging revealed that this was progression of lung cancer, with enlarging mediastinal and hilar lymph nodes.

No QTc prolongation on serial ECGs was detected during the trial (Figure 1A). In contrast to the previously tested agent RTA 402 with a similar triterpenoid-derived scaffold, ${ }^{16}$ no 
Table 2 Omaveloxolone attributable AEs

\begin{tabular}{|c|c|c|c|c|c|c|c|c|c|}
\hline Dose, mg & 2.5 & & 5 & & 10 & & 15 & & Total \\
\hline Grade & I & 2 & 1 & 2 & 1 & 2 & 1 & 2 & \\
\hline \multicolumn{10}{|l|}{ Symptom } \\
\hline Chronic joint pain & I & - & - & - & - & - & - & - & I \\
\hline Nausea or vomiting & $\mathrm{I}$ & - & - & - & - & - & - & - & I \\
\hline Vomiting & I & - & - & - & - & - & - & - & 1 \\
\hline Papular rash, arm and leg & I & - & - & - & - & - & - & - & I \\
\hline Chronic leg swelling & I & - & - & - & - & - & - & - & I \\
\hline Fatigue & I & - & - & - & - & - & - & - & I \\
\hline \multicolumn{10}{|l|}{ Laboratory parameter } \\
\hline Anemia mean & 2 & - & - & - & - & - & - & - & 1 \\
\hline LDH increased & I & - & - & - & - & - & - & - & I \\
\hline ALT increased & - & - & - & - & - & I & - & - & 1 \\
\hline AST increased & - & - & $\mathrm{I}$ & - & - & - & - & - & 1 \\
\hline Alkaline phosphatase increased & - & - & 1 & - & I & - & - & - & 2 \\
\hline
\end{tabular}

Notes: No grade 3 or 4 drug-attributable adverse events were observed. Includes all possible, probable, or definite attributions.

Abbreviations: AEs, adverse events; ALT, alanine aminotransferase; AST, aspartate aminotransferase; LDH, lactate dehydrogenase.

Table 3 All AEs, regardless of attribution

\begin{tabular}{|c|c|c|c|c|c|}
\hline Grade & $\mathbf{I}$ & 2 & 3 & 4 & Total \\
\hline \multicolumn{6}{|l|}{ Symptom } \\
\hline Pain (all types) & 2 & 2 & 2 & - & 6 \\
\hline Cough & 1 & I & - & - & 2 \\
\hline Dyspnea & - & - & 2 & - & 2 \\
\hline Pneumonia & - & - & 2 & - & 2 \\
\hline Vomiting & I & I & - & - & 2 \\
\hline Chronic leg swelling & $\mathrm{I}$ & - & - & - & 1 \\
\hline Constipation & 1 & - & - & - & I \\
\hline Dehydration & 1 & - & - & - & 1 \\
\hline Depression & - & I & - & - & I \\
\hline Dermatitis, scalp & 1 & - & - & - & I \\
\hline Fall & 1 & - & - & - & I \\
\hline Fever & 1 & - & - & - & I \\
\hline Flu-like symptoms & $\mathrm{I}$ & - & - & - & I \\
\hline Laryngeal hemorrhage & I & - & - & - & I \\
\hline Nausea or vomiting & 1 & - & - & - & I \\
\hline Papular rash, arm and leg & 1 & - & - & - & 1 \\
\hline Pericardial effusion & - & I & - & - & I \\
\hline Pulmonary hypertension & - & I & - & - & 1 \\
\hline Wheezing & I & - & - & - & I \\
\hline \multicolumn{6}{|l|}{ Laboratory parameter } \\
\hline Anemia mean & 3 & I & I & - & 5 \\
\hline Alkaline phosphatase increased & 2 & - & - & - & 2 \\
\hline Hypercalcemia & - & I & $\mathrm{I}$ & - & 2 \\
\hline ALT increased & - & I & - & - & I \\
\hline AST increased & - & - & 1 & - & 1 \\
\hline BUN increased & I & - & - & - & I \\
\hline Cholesterol increased & I & - & - & - & I \\
\hline Hypoglycemia & I & - & - & - & I \\
\hline Hypokalemia & - & I & - & - & I \\
\hline Hyponatremia & 1 & - & - & - & 1 \\
\hline LDH increased & I & - & - & - & I \\
\hline Leukocytosis & $\mathrm{I}$ & - & - & - & I \\
\hline Phosphorous increased & 1 & - & - & - & 1 \\
\hline Triglycerides increased & I & - & - & - & I \\
\hline
\end{tabular}

Abbreviations: AEs, adverse events; ALT, alanine aminotransferase; AST, aspartate aminotransferase; BUN, blood urea nitrogen; LDH, lactate dehydrogenase. increase in plasma BNP over normal levels $(<300 \mathrm{pg} / \mathrm{mL})$ was detected (Figure 1B).

\section{Pharmacokinetics}

Pharmacokinetic parameters of omaveloxolone were estimated for all dose cohorts and are summarized in Table 4. Under fasting conditions, omaveloxolone was rapidly absorbed after oral administration, with mean $T_{\text {max }}$ of $2-4$ hours across dose levels. On day 1 , systemic exposures were comparable between the 2.5- and 5-mg dose groups, and higher in the 10and 15-mg dose groups (Figure 2A). Proportional increases in drug exposure $\left(\mathrm{AUC}_{0-t}\right.$ and $\left.C_{\max }\right)$ were observed across the dose range studied (Figure 2B and C). Terminal elimination half-life ranged from 9 hours on day 1 to 20 hours on day 28 , with no apparent dose-dependent trends. Because of the limited number of samples collected over a relatively short interval, the reported values were considered estimates.

Additional samples were collected from three patients who continued dosing beyond the initial 28-day cycle, with collections occurring pre-dose and 2 and 4 hours after administration when possible. Generally, the omaveloxolone concentrations observed in these patients on day 28 of the subsequent cycles were similar to the time-matched samples observed on day 28 during the first cycle.

\section{Pharmacodynamics}

An increase in select Nrf2 target gene expression was observed during the course of treatment, across dose levels (Figure 3A). The interpretation was limited by higher than expected variation among samples in the assay. Likewise, a dose-dependent increase in mRNA expression of select Nrf2 anti-oxidant genes was observed across dose levels (Figure 3B).

Exploratory IHC was assessed from baseline and ontreatment tumor biopsies from two melanoma patients. In a patient from the $10 \mathrm{mg}$ dose level, reductions in expression of NT and iNOS were observed compared to baseline (Figure 4A). This observation was consistent with prior studies, which demonstrated a decrease in reactive nitration in response to treatment with bardoxolone methyl. ${ }^{4}$ However, a patient from the $5 \mathrm{mg}$ dose level had equivocal change in iNOS and NT (Figure 4B). Both of her biopsies were from bone metastases and contained limited tumor material.

In addition, an apparent decrease in monocytic lineage cells was observed during the course of omaveloxolone treatment compared to other cellular compartments (Figure 5). Unfortunately, sufficient PBMCs for more detailed flow cytometry analysis were not collected. Therefore, the reproducibility or significance of this finding remains unclear. 

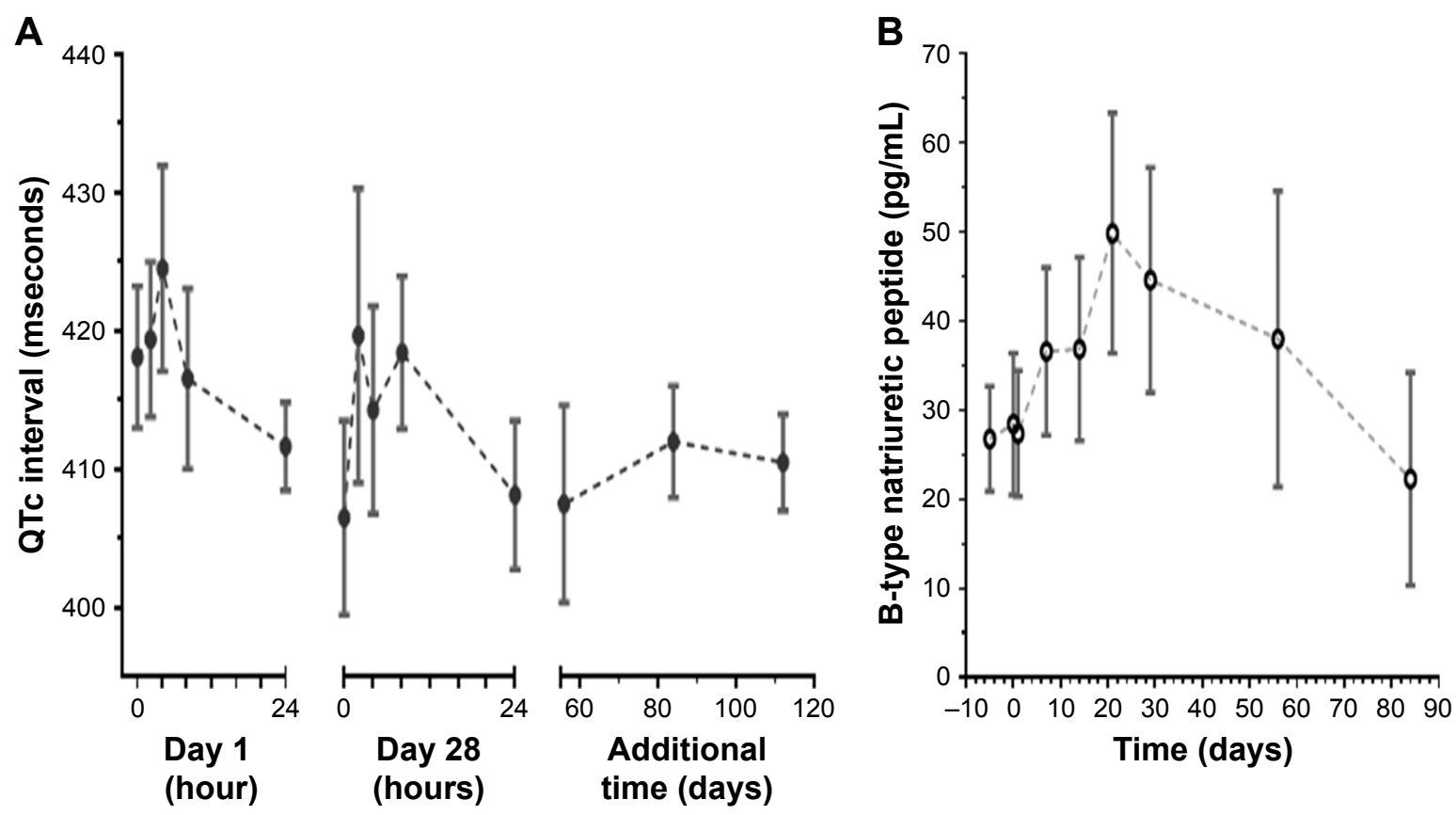

Figure I Safety parameters over time.

Notes: (A) QTc by Frederica's formula during omaveloxolone treatment are shown. Levels did not exceed normal range (<440 mseconds). (B) Overall plasma B-type natriuretic peptide levels during omaveloxolone treatment. Levels did not exceed normal range $(<300 \mathrm{pg} / \mathrm{mL})$. Values for all dose levels $(\mathrm{n}=\mathrm{II})$ are shown. Time 0 represents prior to dose. Circles represent mean with standard error.

Specifically, we could not determine whether these were monocytes or possibly monocytic suppressor cells. It is conceivable that this was a treatment effect or alternatively due to the effect of metastatic cancer.

\section{Survival outcomes}

Median progression-free survival was 1.5 months (95\% CI 1.0-1.9), and all subjects experienced progression (Figure 6A).

Table 4 Omaveloxolone pharmacokinetics

\begin{tabular}{|c|c|c|c|c|c|c|c|c|}
\hline Dose, mg & 2.5 & & 5 & & 10 & & 15 & \\
\hline Day & I & 28 & I & 28 & 1 & 28 & I & 28 \\
\hline Evaluable (n) & 3 & 3 & 3 & 3 & 3 & $3 *$ & $2^{* *}$ & $\mathrm{n} / \mathrm{a}$ \\
\hline \multicolumn{9}{|c|}{$\mathrm{AUC}_{0-\mathrm{t}}$ (hours $\mathrm{ng} / \mathrm{mL}$ ) } \\
\hline Mean & 27.6 & 44.8 & 31.6 & 63.7 & 114.4 & 188.3 & 183.5 & - \\
\hline SD & 32.7 & 51.5 & 7.9 & 17.0 & 141.7 & 176.7 & 27.6 & - \\
\hline \multicolumn{9}{|l|}{$T_{\max }$ (hours) } \\
\hline Mean & 3.3 & 2.9 & 2.3 & 2.5 & 2.0 & 3.0 & 3.5 & - \\
\hline SD & 2.1 & I & 0.6 & 1.2 & 1.5 & - & 2.8 & - \\
\hline \multicolumn{9}{|l|}{$C_{\max }(\mathrm{ng} / \mathrm{mL})$} \\
\hline Mean & 4.6 & 4.5 & 3.9 & 5.6 & 15.7 & 13.4 & 16.6 & - \\
\hline SD & 7.8 & 4.6 & 2.1 & 1.8 & 34.5 & 9.7 & 6.9 & - \\
\hline \multicolumn{9}{|l|}{$T_{1 / 2}$ (hours) } \\
\hline Mean & 10.2 & 21.2 & 8.9 & 19.7 & 9.2 & 19.8 & 8.7 & - \\
\hline SD & 2.4 & 20.1 & 1.3 & 8.1 & 0.6 & 4.1 & 3.7 & - \\
\hline
\end{tabular}

Notes: Averages represent geometric mean. *One tube received broken at day 28. **Dose level completed in two subjects.

Abbreviations: $\mathrm{AUC}_{0-t}$, area under curve from time 0 to last; $\mathrm{n} / \mathrm{a}$, no samples available.
Consistent with the poor prognosis of the patients enrolled, median overall survival was 5.8 months $(95 \%$ CI 2.6-8.9), after nine of 11 events and 27 months of median follow-up. All deaths were attributable to progressive cancer. No confirmed radiologic responses were observed. One subject with lung cancer had stable disease lasting more than 12 months (Figure 6B). Although her target lesion measurements did decrease, the reports consistently included the caveat that the two target lesions were difficult to measure due to lung atelectasis.

\section{Discussion}

In this first-in-human dose-escalation Phase I study, we demonstrate the safety of omaveloxolone in patients with advanced solid tumors within a small sample size of patients. Omaveloxolone was tolerated at doses observed to augment Nrf2 signaling in peripheral cells and showed fairly doseproportional pharmacokinetics. The most common drugrelated toxicities included grade 1 anemia and alkaline phosphatase elevation. Because the MTD of oral omaveloxolone was not reached, it is conceivable that the omaveloxolone dose could be further increased. Nonetheless, the suggestion of target modulation at relatively low doses indicated that the 5-10 $\mathrm{mg}$ dose was sufficient to serve as a starting point for dose escalation in subsequent combination 

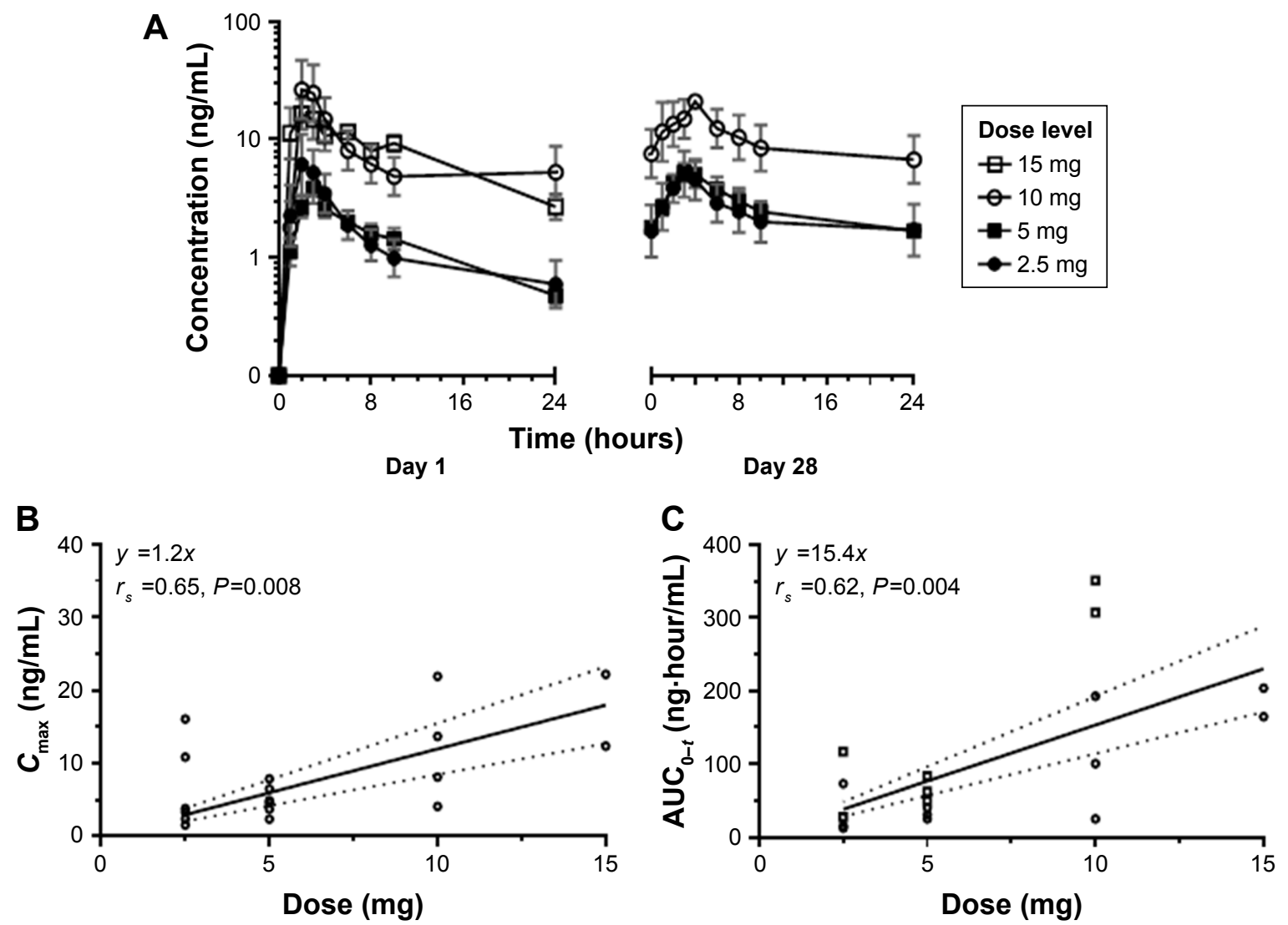

Figure 2 Pharmacokinetic assessment of omaveloxolone.

Notes: (A) Drug concentration over time at days I and 28. Day 28 collections were missing for I5 mg cohort. (B) Maximum drug concentration in plasma. (C) Area under the plasma concentration-time curve was plotted against dose using linear regression for both day I and 28 . Dotted lines represent $90 \%$ confidence interval. $r$, Spearman's rho correlation coefficient with two-sided significance.

trials. Currently, omaveloxolone is being further studied in a dose-escalation trial in combination with nivolumab or ipilimumab for solid tumors, which started at the $5 \mathrm{mg}$ dose level. ${ }^{17}$

This Phase I trial was characterized by important limitations. Unfortunately, optional tumor biopsies were not performed for most patients, and thus, we have no consistent evidence showing modulation of antioxidant activity within the tumor. Due to the initiation of a competing combination trial for solid tumors, the dose escalation was concluded at $15 \mathrm{mg}$, before a definitive escalation to MTD could be reached. ${ }^{17}$ Therefore, all statements regarding safety must be moderated by the small sample size of this trial. Although this trial had a small sample size, no adverse safety signal was observed. Another limitation is that most subjects progressed within several months of treatment, and mean drug exposure was only 85 days. Therefore, no conclusive assessment of long-term toxicity was possible. This inevitable disease progression was not unexpected, since all subjects had progressive and refractory cancer at baseline. Omaveloxolone is also being further explored in non-cancer populations in Phase II trials for mitochondrial disorders (Table 5). ${ }^{18,19}$

Omaveloxolone is a second-generation analog of bardoxolone methyl, whose development was temporarily halted by a higher-than-expected number of diastolic heart failure events in a Phase III registration trial for advanced CKD. ${ }^{20}$ It was subsequently determined that doses of bardoxolone methyl at $20 \mathrm{mg}$ /day promoted fluid retention in a subset of CKD patients with preexisting risk factors, including prior hospitalization for heart failure and elevated BNP at baseline. Moreover, a cumulative increase in BNP within 4 weeks of treatment with bardoxolone methyl was observed in advanced CKD patients. This phenomenon was likely due to suppression of basal endothelin-1 secretion and endothelin receptor A expression, whereby acute modulation of this pathway in advanced CKD patients' population likely promoted acute sodium retention which translated into frank fluid overload in susceptible patients. ${ }^{16}$ Importantly, no cumulative increase in BNP was observed with omaveloxolone during this trial 

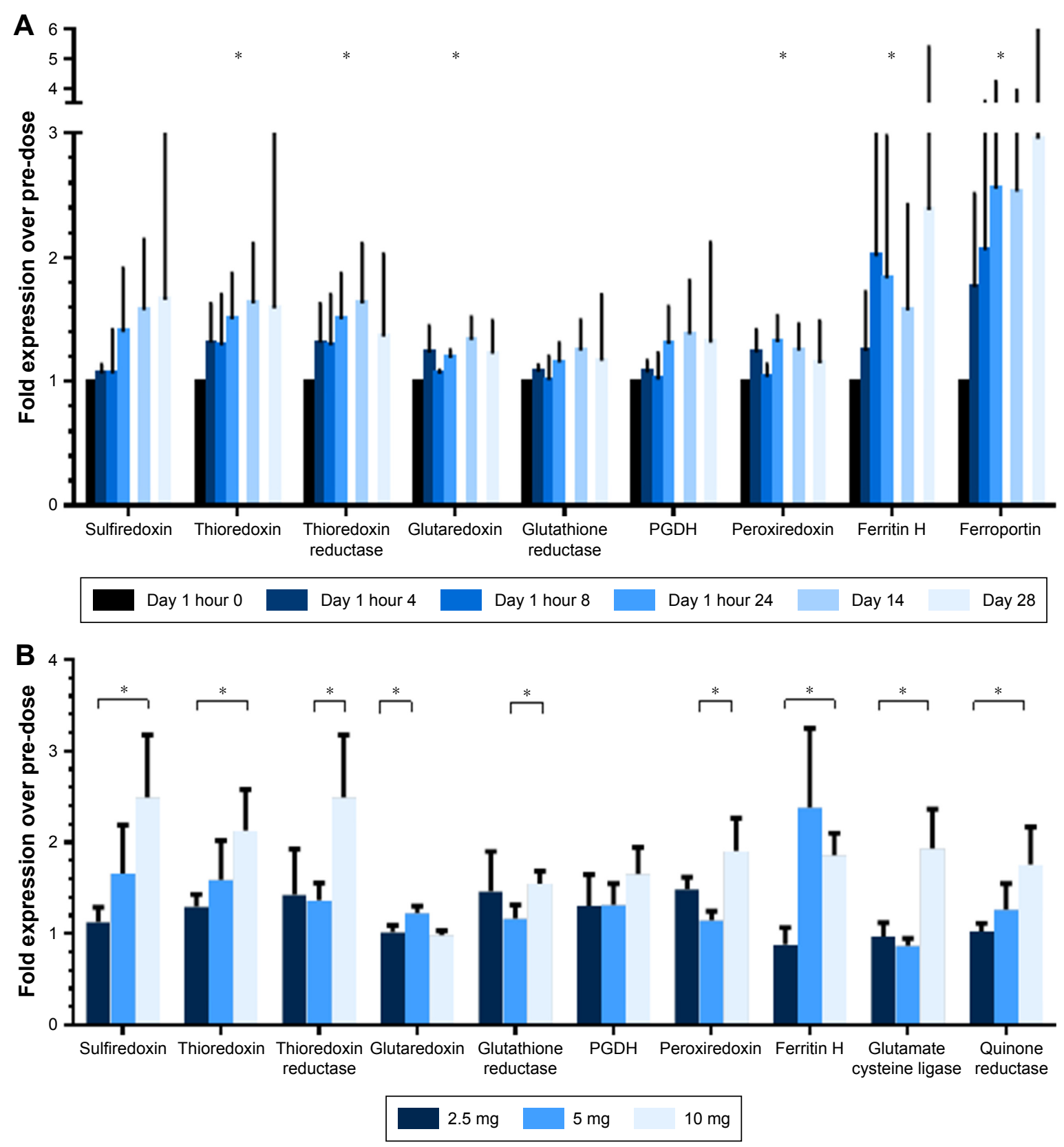

Figure 3 Effect of continuous oral omaveloxolone upon Nrf2 target genes.

Notes: mRNA expression was normalized to two housekeeping genes and then pre-dose level. (A) Expression over timepoints, $5 \mathrm{mg}$ cohort example ( $\mathrm{n}=3$ ). Bars represent mean with standard error. $* P<0.05$ for Wilcoxon signed-rank test of whether post-dose timepoints exceeded normalized pre-dose level of I. (B) Expression by dose level for samples collected at day $28, n=3$ for each cohort. Bars represent mean with standard error. $* P<0.05$ for one-sided $t$-test for comparison between indicated groups. Abbreviations: Ferritin H, ferritin heavy subunit; mRNA, messenger RNA; Nrf2, nuclear factor erythroid 2-related factor 2; PGDH, prostaglandin dehydrogenase I.

(Figure 1B). Moreover, in a subsequent Phase II trial for pulmonary arterial hypertension, doses of bardoxolone methyl as high as $10 \mathrm{mg} /$ day improved exercise performance compared to placebo without evidence of fluid retention. ${ }^{21}$ Therefore, it is possible that the MTD of triterpenoid analogs may exceed the dose required to demonstrate biologic activity.

Although pharmacodynamic activity was seen, no objective responses were observed in our trial. In contrast, clinical activity was observed in one lymphoma patient and one thyroid cancer patient in a trial of bardoxolone methyl. ${ }^{22}$ This discordance may be related to the histology of cancers in our trial limited to melanoma and lung cancer only. ${ }^{23,24}$ MDSCs are implicated in tumor cell resistance to adoptive T-cell therapies, and inhibition of MDSC oxidative pathways may restore anti-tumor activity. ${ }^{25,26}$ Peripheral MDSCs are associated with failure to respond to dual checkpoint 


\section{A}
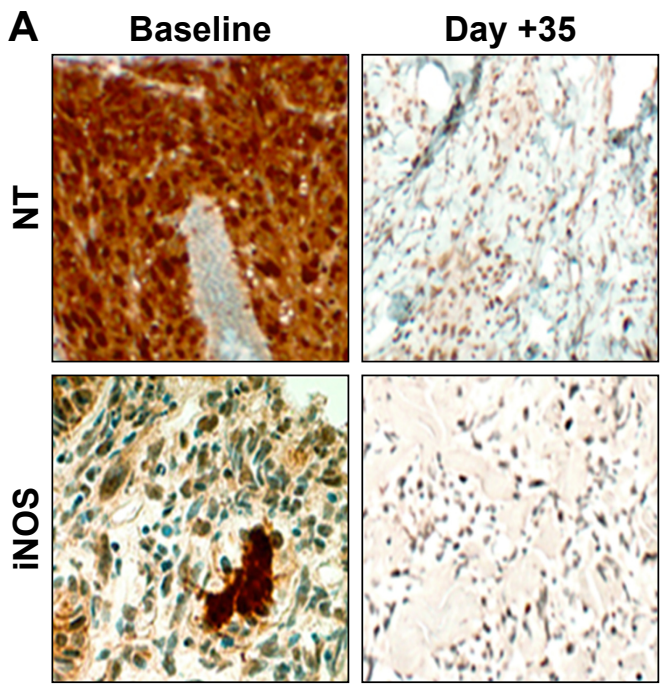

B Baseline
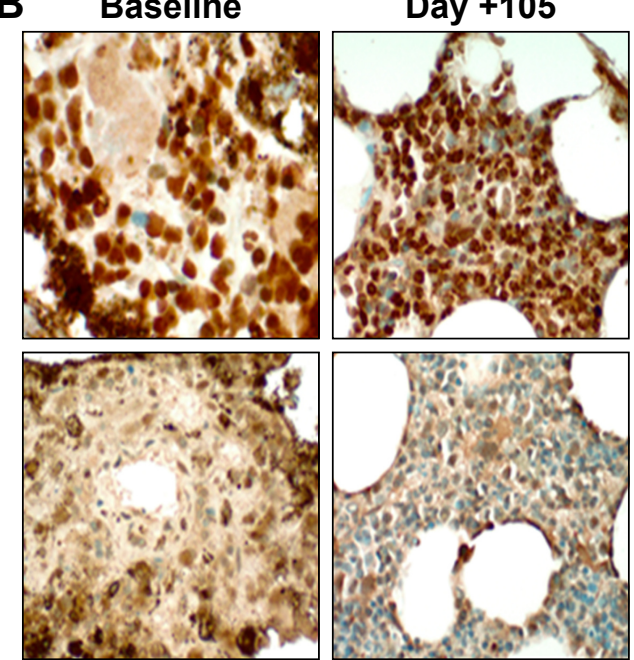

Figure 4 Representative images of NT and iNOS from limited on-treatment tumor biopsies for two patients with metastatic melanoma.

Notes: (A) Punch skin biopsy at day +35 from patient in $10 \mathrm{mg}$ cohort showed overall decrease in expression of nitration markers compared to baseline. (B) Bone metastasis biopsy at day +105 from patient in $5 \mathrm{mg}$ cohort showed little difference in NT or iNOS expression compared to baseline. Archival bone samples were difficult to evaluate due to prior decalcification and scant tumor. Serial biopsies for additional patients were not available. Original magnification $4 \times$.

Abbreviations: iNOS, inducible isoform of nitric oxide synthase; NT, nitrotyrosine.

blockade against CTLA-4 and PD-1 in advanced melanoma and were associated with failure to respond to nivolumab monotherapy. ${ }^{27,28}$ In addition, the MDSCs of non-responders to ipilimumab may produce more nitric oxide than those of responders. ${ }^{29}$ Moreover, cytokine-directed abrogation of iNOS from cultured ex vivo MDSCs was sufficient to restore T-cell proliferation and inhibit tumor cell growth in vitro. ${ }^{30}$ Therefore, it is conceivable that omaveloxolone may be

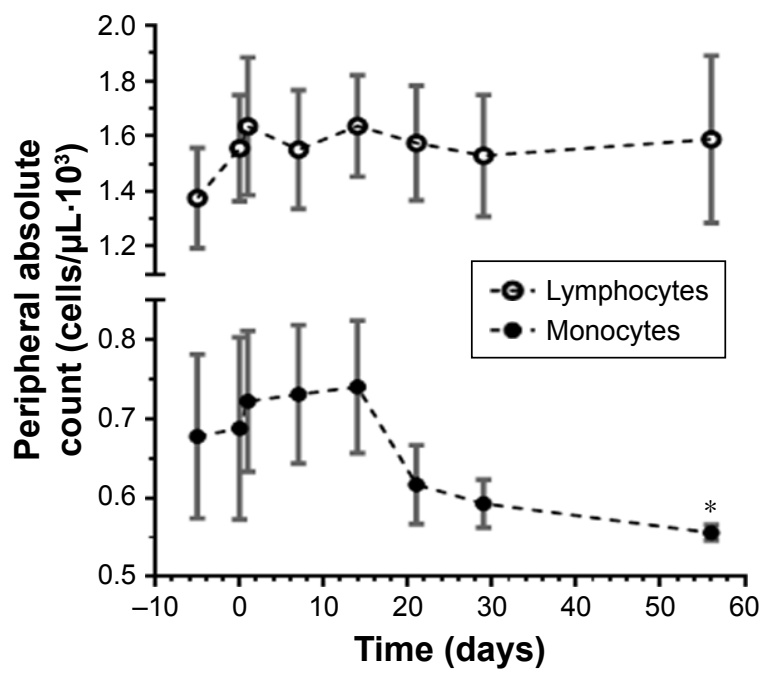

Figure 5 Decrease in peripheral blood monocytic cells over time.

Notes: Absolute peripheral cell counts by automated differential during omaveloxolone treatment for all dose levels $(n=I I)$ are shown. Time 0 represents first day of dosing. Circles represent mean with standard error. *Denotes $P=0.05$ for one-sided $t$-test compared to baseline. a favorable combination partner for immune checkpoint blockade of solid tumors.

Alternative strategies to relieve MDSC-mediated tumor immunosuppression include blockade of colony-stimulating factor-1 (CSF-1R) and interleukin-34 signaling via its receptor, CSF-1R. For example, the selective CSF-1R inhibitor BLZ945 reduced the activity of MDSCs and slowed tumor progression in a murine neuroblastoma model and is being tested in Phase I/II trials. ${ }^{31}$ Similarly, neutralizing antibodies to chemokine receptors required for MDSC recruitment, such as CCR2 (C-C chemokine receptor type 2) and CXCR4 (C-X-C chemokine receptor type 4), are being evaluated for solid tumors. $^{32}$ By promoting major histocompatibility complex II expression on myeloid cells, CD40 agonists can also enhance antigen presentation and subsequent anti-tumor responses..$^{33,34}$ Likewise, antagonism of vascularization via angiopoietin receptor (tyrosine kinase with immunoglobulin-like and EGFlike domains 2) or vascular endothelial growth factor receptor inhibitors may induce differentiation of MDSCs into immunocompetent myeloid cells and reduce tumor growth. ${ }^{35,36}$ Thus, semisynthetic triterpenoids are only one of many expanding drug classes intended to abrogate MDSC-associated immunosuppression. Evidence indicates that omaveloxolone induction of Nrf2 target genes can potentially improve muscle function, oxidative phosphorylation, and antioxidant capacity in patients with mitochondrial myopathies. ${ }^{37}$ Therefore, this Phase I trial represents a first step toward defining the safety of a new drug with potential in multiple disease types. ${ }^{38}$ 


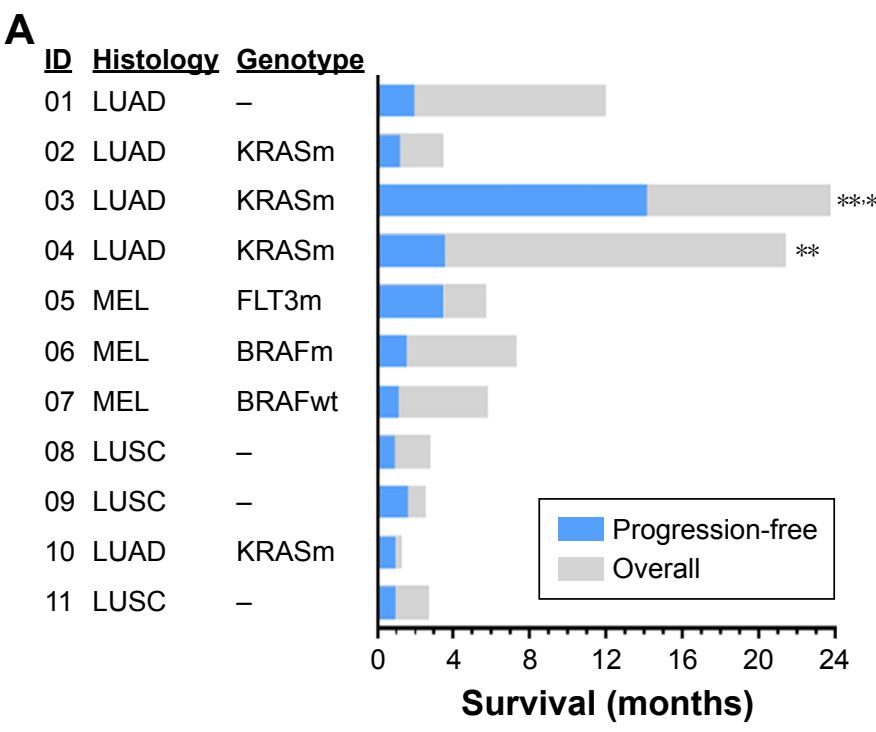

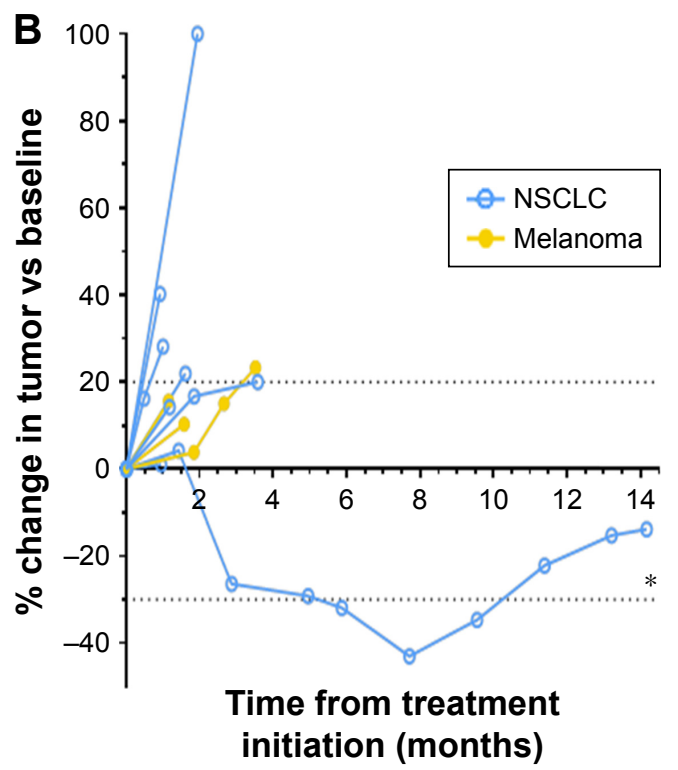

Figure 6 Patient outcome after treatment.

Notes: (A) Swimmers plot of survival for omaveloxolone-treated subjects according to modified RECIST. Numbers indicate dose level, in mg. (B) Spider plot showing change in target lesions by modified RECIST. *Target lesion noted as difficult to measure due to atelectasis. **Still alive during last assessment.

Abbreviations: LUSC, squamous lung cancer; LUAD, lung adenocarcinoma; MEL, melanoma; NSCLC, non-small cell lung cancer; RECIST, response evaluation criteria in solid tumor.

\section{Conclusion}

Our findings suggest that omaveloxolone is well tolerated by patients with advanced solid tumors, at doses which may activate the Nrf2 pathway in the PBMC compartment. The safety and pharmacology profile from this small trial supports the continued clinical investigation of omaveloxolone for humans.

\section{Abbreviations}

AEs, adverse events; $\mathrm{AUC}_{0-t}$, area under the plasma concentration-time curve from time zero to the time $(t)$ of the last measurable concentration; BNP, B-type natriuretic peptide; $\mathrm{C}_{\max }$, maximum concentration; CCR2, $\mathrm{C}-\mathrm{C}$ chemokine receptor type 2 ; $\mathrm{CD}$, cluster of differentiation; CKD, chronic kidney disease; CSF-1R, colony-stimulating factor-1 receptor; CTCAE, common toxicity criteria for adverse events; CTLA-4, cytotoxic T-lymphocyte-associated protein 4; CXCR4, C-X-C chemokine receptor type 4; DLT, dose-limiting toxicity; ECG, electrocardiogram; ECOG, Eastern Cooperative Oncology group; ICH-GCP, International Conference on Harmonization Good Clinical Practice; IHC, immunohistochemistry; iNOS, inducible isoform of nitric oxide synthase; MDSCs, myeloid-derived suppressor cells; mRNA, messenger RNA; MTD, maximum tolerated dose; Nrf2, nuclear factor erythroid 2-related factor 2; NT, nitrotyrosine; PBMCs, peripheral blood mononuclear cells; PD-1, programmed cell death protein 1; RECIST, response evaluation criteria in solid tumor; ROS, reactive oxygen species; RPL13A, 60S ribosomal protein L13a; $r_{s}$, Spearman's rho correlation coefficient; $t^{1 / 2}$, terminal elimination half-life; $T_{\max }$, time of maximum concentration; ULN, upper limit of normal.

Table 5 Current clinical investigation of omaveloxolone

\begin{tabular}{|c|c|c|c|c|c|c|}
\hline Trial & Indication & Size & Form & Phase & Start date & NCT\# \\
\hline MOTOR & Treatment of mitochondrial myopathy & 56 & Oral & 2 & $4-2015$ & 02255422 \\
\hline MOXle & Treatment of Friedrich's ataxia & 56 & Oral & 2 & $1-2015$ & 02255435 \\
\hline PRIMROSE & Radiation dermatitis in adjuvant breast cancer & 187 & Lotion & 2 & $6-2014$ & 02142959 \\
\hline REVEAL* & Treatment of metastatic melanoma & 84 & Oral & $\mathrm{I} / 2$ & $10-20 \mid 4$ & 02259231 \\
\hline GUARD & Prevention of post-operative corneal endothelial loss & 304 & Suspension & 2 & $5-2014$ & 02128113 \\
\hline
\end{tabular}

Note: *Combination trial of omaveloxolone with nivolumab or ipilimumab.

Abbreviation: NCT, national clinical trials identifier number. 


\section{Acknowledgments}

This work has been supported, in part, by the Tissue Core Facility at the H. Lee Moffitt Cancer Center \& Research Institute, an NCI designated Comprehensive Cancer Center (P30-CA0,76,292). We gratefully acknowledge Drs Theresa Boyle, Alberto Chiappori, and Milijana Ugrenovic for their feedback on the manuscript and contributions toward the trial.

This trial was supported by Reata Pharmaceuticals, Inc and Abbvie, Inc. Correlative studies were supported by 2013 Conquer Cancer Foundation Young Investigator Award (to BCC), NCI P50 CA1 19997 (to EBH). Apart from the authors themselves, the funding organizations had no direct role in the preparation, review, or approval of the manuscript.

\section{Availability of data and material}

The data that support the findings of this study are not publically available due to a third party restriction and confidentiality disclosure agreement with Reata Pharmaceuticals, Inc.

\section{Ethics approval and consent to participate}

All participants provided written informed consent. The study was approved by Liberty IRB; IRB00008679.

\section{Author contributions}

All authors contributed toward data analysis, drafting and critically revising the paper and agree to be accountable for all aspects of the work.

\section{Disclosure}

The authors MPC, SAR, JWP, and CJM are employed by, and have a financial interest in, Reata Pharmaceuticals. DIG has served as a paid consultant with Reata Pharmaceuticals. MDM is employed by, and has a financial interest in, AbbVie, Inc. The author BCC has served as a consultant/advisory role for AbbVie, Inc in 2017. The authors report no other conflicts of interest in this work.

\section{References}

1. Nagaraj S, Gupta K, Pisarev V, et al. Altered recognition of antigen is a mechanism of CD8+ T cell tolerance in cancer. Nature Med. 2007; 13(7):828-835.

2. Hiramoto K, Satoh H, Suzuki T, et al. Myeloid lineage-specific deletion of antioxidant system enhances tumor metastasis. Cancer Prev Res. 2014;7(8):835-844.

3. Sawant A, Schafer CC, Jin TH, et al. Enhancement of antitumor immunity in lung cancer by targeting myeloid-derived suppressor cell pathways. Cancer Res. 2013;73(22):6609-6620.
4. Nagaraj S, Youn JI, Weber H, et al. Anti-inflammatory triterpenoid blocks immune suppressive function of myeloid-derived suppressor cells and improves immune response in cancer. Clin Cancer Res. 2010; 16(6):1812-1823.

5. Huerta C, Jiang X, Trevino I, et al. Characterization of novel smallmolecule NRF2 activators: Structural and biochemical validation of stereospecific KEAP1 binding. Biochim Biophys Acta. 2016; 1860(11 Pt A):2537-2552.

6. Cleasby A, Yon J, Day PJ, et al. Structure of the BTB domain of Keap1 and its interaction with the triterpenoid antagonist CDDO. PLoS One. 2014;9(6):e98896

7. Stewart J, Hengstler J, Bolt H. Control of oxidative stress by the Keap1Nrf2 pathway. Arch Toxicol. 2011;85(4):239.

8. Probst BL, Trevino I, McCauley L, et al. RTA 408, a novel synthetic triterpenoid with broad anticancer and anti-inflammatory activity. PLoS One. 2015;10(4):e0122942.

9. Alexeev V, Lash E, Aguillard A, et al. Radiation protection of the gastrointestinal tract and growth inhibition of prostate cancer xenografts by a single compound. Mol Cancer Ther. 2014;13(12) 2968-2977.

10. Goldman DC, Alexeev V, Lash E, Guha C, Rodeck U, Fleming WH. The triterpenoid RTA 408 is a robust mitigator of hematopoietic acute radiation syndrome in mice. Radiat Res. 2015;183(3):338-344.

11. Reisman SA, Lee C-YI, Meyer CJ, Proksch JW, Sonis ST, Ward KW. Topical application of the synthetic triterpenoid RTA 408 protects mice from radiation-induced dermatitis. Radiat Res. 2014;181(5): 512-520.

12. Reisman SA, Lee CY, Meyer CJ, Proksch JW, Ward KW. Topical application of the synthetic triterpenoid RTA 408 activates Nrf2 and induces cytoprotective genes in rat skin. Arch Dermatol Res. 2014;306(5): 447-454.

13. Reisman SA, Goldsberry AR, Lee CY, et al. Topical application of RTA 408 lotion activates Nrf2 in human skin and is well-tolerated by healthy human volunteers. BMC Dermatol. 2015;15(1):10.

14. Liu X, Ward K, Xavier C, et al. The novel triterpenoid RTA 408 protects human retinal pigment epithelial cells against $\mathrm{H} 2 \mathrm{O}$ 2-induced cell injury via NF-E2-related factor 2 (Nrf2) activation. Redox Biol. 2016;8:98-109.

15. Eisenhauer E, Therasse P, Bogaerts J, et al. New response evaluation criteria in solid tumours: revised RECIST guideline (version 1.1). Eur J Cancer. 2009;45(2):228-247.

16. Chin MP, Reisman SA, Bakris GL, et al. Mechanisms contributing to adverse cardiovascular events in patients with type 2 diabetes mellitus and stage 4 chronic kidney disease treated with bardoxolone methyl. Am J Nephrol. 2014;39(6):499-508.

17. Reata Pharmaceuticals, Inc. RTA 408 Capsules in patients with melanoma-REVEAL; 2017. Available from: https://clinicaltrials.gov/ ct2/show/NCT02259231. NLM identifier NCT02259231. Accessed March 6, 2017.

18. Reata Pharmaceuticals, Inc. RTA 408 Capsules in patients with mitochondrial myopathy - MOTOR; 2017. Available from: https://clinicaltrials.gov/ct2/show/NCT02255422. NLM identifier NCT02255422. Accessed March 6, 2017.

19. Reata Pharmaceuticals, IncRTA 408 Capsules in patients with Friedreich's ataxia - MOXIe; NLM identifier. NCT02255435. Available from: https:/clinicaltrials.gov/ct2/show/NCT02255435. Accessed March 6, 2017.

20. Tayek JA, Kalantar-Zadeh K. The extinguished BEACON of bardoxolone: not a Monday morning quarterback story. Am J Nephrol. 2013 37(3):208-211.

21. Oudiz RJ, Meyer CJ, Melanie C, et al. Initial Data Report from "LARIAT": A Phase 2 Study of Bardoxolone Methyl in PAH Patients on Stable Background Therapy. Chest. 2015;148(4: Meeting Abstracts):639A.

22. Hong DS, Kurzrock R, Supko JG, et al. A phase I first-in-human trial of bardoxolone methyl in patients with advanced solid tumors and lymphomas. Clin Cancer Res. 2012;18(12):3396-3406. 
23. Speranza G, Gutierrez ME, Kummar S, et al. Phase I study of the synthetic triterpenoid, 2-cyano-3, 12-dioxoolean-1, 9-dien-28-oic acid (CDDO), in advanced solid tumors. Cancer Chemother Pharmacol. 2012;69(2): 431-438.

24. Tsao T, Kornblau S, Safe S, et al. Role of peroxisome proliferatoractivated receptor- $\gamma$ and its coactivator DRIP205 in cellular responses to CDDO (RTA-401) in acute myelogenous leukemia. Cancer Res. 2010;70(12):4949-4960.

25. Hossain F, Al-Khami AA, Wyczechowska D, et al. Inhibition of fatty acid oxidation modulates immunosuppressive functions of myeloidderived suppressor cells and enhances cancer therapies. Cancer Immunol Res. 2015;3(11):1236-1247.

26. Shen L, Sundstedt A, Ciesielski M, et al. Tasquinimod modulates suppressive myeloid cells and enhances cancer immunotherapies in murine models. Cancer Immunol Res. 2015;3(2):136-148.

27. Callahan MK, Horak CE, Curran MA, et al. Peripheral and tumor immune correlates in patients with advanced melanoma treated with combination nivolumab (anti-PD-1, BMS-936558, ONO-4538) and ipilimumab. Journal of Clinical Oncology. 2013;31(15):3003.

28. Weber J, Gibney G, Kudchadkar R, et al. Phase I/II study of metastatic melanoma patients treated with nivolumab who had progressed after ipilimumab. Cancer Immunol Res. 2016;4(4):345-353.

29. Gebhardt C, Sevko A, Jiang H, et al. Myeloid cells and related chronic inflammatory factors as novel predictive markers in melanoma treatment with ipilimumab. Clin Cancer Ress. 2015;21(24): 5453-5459.

30. Jayaraman P, Parikh F, Krupar R, Parihar R, Varier I, Sikora A. Abstract PR005: TGF-betal primed myeloid derived suppressor cells decrease tumor growth and lose their ability to inhibit $\mathrm{T}$ cell proliferation via iNOS downregulation. Cancer Immunol Res. 2016;4(Suppl 1): PR005-PR005.
31. Mao Y, Eissler N, Le Blanc K, Johnsen JI, Kogner P, Kiessling R. Targeting suppressive myeloid cells potentiates checkpoint inhibitors to control spontaneous neuroblastoma. Clin Cancer Res. 2016;22(15): 3849-3859.

32. Vela M, Aris M, Llorente M, Garcia-Sanz JA, Kremer L. Chemokine receptor-specific antibodies in cancer immunotherapy: achievements and challenges. Fronts Immunol. 2015;6:12.

33. Beatty GL, Chiorean EG, Fishman MP, et al. CD40 agonists alter tumor stroma and show efficacy against pancreatic carcinoma in mice and humans. Science. 2011;331(6024):1612-1616.

34. Dahan R, Barnhart BC, Li F, Yamniuk AP, Korman AJ, Ravetch JV. Therapeutic activity of agonistic, human anti-CD40 monoclonal antibodies requires selective Fc $\gamma \mathrm{R}$ engagement. Cancer Cell. 2016;29(6): $820-831$.

35. Ibberson M, Bron S, Guex N, et al. TIE-2 and VEGFR kinase activities drive immunosuppressive function of TIE-2-expressing monocytes in human breast tumors. Clin Cancer Res. 2013;19(13):3439-3449.

36. Panka DJ, Arbeit RD, Mier JW. Regulation of MDSC trafficking and function in RCC by CXCR4 in the presence of a VEGF-R antagonist. AACR. 2016;76(14): Abstract nr 4155.

37. Dinkova-Kostova AT, Baird L, Holmström KM, Meyer CJ, Abramov AY. The spatiotemporal regulation of the Keap1-Nrf2 pathway and its importance in cellular bioenergetics. Biochem Soc Trans. 2015;43(4): 602-610.

38. Liby KT, Sporn MB. Synthetic oleanane triterpenoids: multifunctional drugs with a broad range of applications for prevention and treatment of chronic disease. Pharmacol Rev. 2012;64(4):972-1003.
OncoTargets and Therapy

\section{Publish your work in this journal}

OncoTargets and Therapy is an international, peer-reviewed, open access journal focusing on the pathological basis of all cancers, potential targets for therapy and treatment protocols employed to improve the management of cancer patients. The journal also focuses on the impact of management programs and new therapeutic agents and protocols on

\section{Dovepress}

patient perspectives such as quality of life, adherence and satisfaction The manuscript management system is completely online and includes a very quick and fair peer-review system, which is all easy to use. Visit http://www.dovepress.com/testimonials.php to read real quotes from published authors. 